\section{Journal of \\ Epilepsy and \\ Clinical \\ Neurophysiology}

J Epilepsy Clin Neurophysiol 2010;16(2):51-58

\title{
Epilepsia Mioclônica Juvenil: \\ Endofenótipos distintos considerando aspectos neuropsicológicos, traços de personalidade e variáveis clínicas*
}

\author{
Sylvie Moschetta**, Daniel Fuentes***, Lia Arno Fiore**, Kette Dualibi Ramos Valente** \\ Instituto de Psiquiatria do Hospital das Clínicas da Faculdade de Medicina da USP
}

\begin{abstract}
RESUMO
Introdução: Estudos neuropsicológicos sugerem que indivíduos com EMJ apresentam disfunção executiva além de maior impulsividade, refletindo uma disfunção de lobo frontal. Este estudo teve como objetivos verificar: 1. o desempenho e gravidade de comprometimento das funções executivas; 2 . presença de traços de personalidade impulsivos; 3 . a correlação entre o desempenho nas funções cognitivas e a expressão de traços de personalidade impulsivos e; 4 . se as variáveis da epilepsia se correlacionam com as disfunções executivas e a um pior controle de impulsos. Métodos: Foram avaliados 42 pacientes com EMJ e 42 sujeitos saudáveis pareados por idade, escolaridade e nível socioeconômico, através de bateria compreensiva de testes neuropsicológicos avaliadores de funções executivas e questionário padronizado de avaliação de traços de personalidade (ITC). Resultados: Pacientes com EMJ tiveram pior desempenho nos testes de atenção imediata, seletiva e sustentada; controle mental e inibitório; flexibilidade mental; fluência verbal; formação de conceitos e manutenção de metas. A disfunção executiva foi moderada/grave em 83,33\%. Pacientes com EMJ apresentaram maior expressão dos traços de personalidade impulsivos. A disfunção executiva se correlacionou com o pior controle dos impulsos. Pacientes refratários apresentaram pior disfunção executiva e maior expressão de traços impulsivos. Conclusões: Nosso estudo demonstra a presença de disfunção atencional e executiva nos pacientes com EMJ, além da presença de traços de personalidade impulsivos. Além disso, verificamos a existência de dois grupos distintos de pacientes, sendo que pacientes mais refratários apresentam-se globalmente comprometidos. Estes achados sugerem que há uma necessidade de melhor caracterização fenotípica dos pacientes com EMJ a fim de incluir endofenótipos visto que nossos resultados demonstram uma possível existência de grupos distintos de pacientes com EMJ.
\end{abstract}

Descritores: Epilepsia mioclônica juvenil; neuropsicologia; personalidade.

\begin{abstract}
Juvenile myoclonic epilepsy: distinct phenotypes considering aspects neuropsychological, personality traits and variables clinics

Introduction: Neuropsychological studies suggest that patients with JME have executive dysfunction and impulsiveness, reflecting a possible frontal lobe dysfunction. This study aimed to verify: 1. the performance and severity of attentional and executive functions; 2 . presence of impulsive personality traits; 3 . correlation between cognitive performance and the expressions of impulsive personality traits; 4 . correlation between epilepsy variables and neuropsychological performance as well as worse impulse control. Methods: We evaluated 42 patients with JME and a group of 42 control subjects, matched for age, education and socioeconomic status with a comprehensive battery of neuropsychological tests of attentional and executive functions and a standardized assessment of personality traits (TCI). Results: Patients with JME showed worse performance than controls on tests of attentional span, working memory, inhibitory control, concept formation, maintenance of goals, and verbal fluency. Executive dysfunction was severe/moderate in $83.33 \%$. Patients with JME showed
\end{abstract}

\footnotetext{
* Trabalho premiado com o Prêmio Cesare Lombroso durante o XXXIII Congresso Brasileiro de Epilepsia, 03-06 junho 2010 (Brasília/DF).

** Laboratório de Neurofisiologia Clínica do Instituto de Psiquiatria do Hospital das Clínicas da Faculdade de Medicina da USP.

*** Serviço de Psicologia e Neuropsicologia do Instituto de Psiquiatria do Hospital das Clínicas da Faculdade de Medicina da USP. Received Apr. 30, 2010; accepted June 03, 2010.
} 
higher expression of personality traits associated with an impaired impulse control. Attentional/executive dysfunction was correlated with poor impulse control. Refractory patients had worse executive dysfunction with an even a greater presence of impulsive personality traits. Conclusions: Our study demonstrates the presence of attentional and executive dysfunction in patients with JME, as well as the presence of impulsive personality traits. We also note the existence of two distinct groups of patients, were more refractory patients appear to present broader impairment. These findings suggest that there is a need for better phenotypic characterization of patients with JME to include diverse phenotypes since our results suggest a possible existence of distinct groups of patients with JME.

Keywords: Juvenile myoclonic epilepsy; neuropsychology; personality.

\section{INTRODUÇÃO}

A Epilepsia Mioclônica Juvenil (EMJ) é uma epilepsia generalizada idiopática geneticamente determinada, caracterizada por crises generalizadas, com início na adolescência, de fácil controle medicamentoso, mas com duração permanente, geralmente associada à ausência de alterações estruturais. ${ }^{1}$

Entretanto, estudos neuropsicológicos sugerem que indivíduos com EMJ têm desempenho prejudicado em vários testes que avaliam funções cognitivas associadas ao funcionamento dos lobos frontais ${ }^{2-6}$ apresentando baixo desempenho especialmente nos testes que requerem formação de conceitos, flexibilidade mental, atenção focal e controle inibitório de respostas.

A maior parte destes estudos não faz uso de uma bateria ampla que avalie as funções executivas e atencionais destes pacientes. As funções executivas e atencionais compreendem diversas funções associadas ao funcionamento dos lobos frontais, como fluência e flexibilidade mental, memória operativa, planejamento, formação de conceitos, controle inibitório de estímulos e os próprios processos de atenção. ${ }^{7-9}$ Portanto, as funções executivas e atencionais não podem ser mensuradas em sua totalidade por um grupo restrito de testes.

Levando em consideração os achados de disfunção do lobo frontal em pacientes com EMJ, pressupõe-se a importância de uma avaliação das funções executivas e atencionais em suas diferentes esferas, a partir de uma bateria neuropsicológica mais extensa.

De maneira complementar, alterações de personalidade e distúrbios psiquiátricos em pacientes com EMJ vêm sendo descritos. ${ }^{10-16} \mathrm{O}$ mais comumente observado é a presença de um pior controle de impulsos, além de irresponsabilidade, autointeresse, negligência dos deveres, instabilidade emocional, exageros, geniosidade e distratibilidade. ${ }^{10-16}$

Os transtornos psiquiátricos e de personalidade de pacientes com EMJ baseiam-se em observações clínicas ${ }^{10,12}$ ou através de instrumentos baseados na classificação categórica baseada nos critérios do DSM-IV. ${ }^{13-15}$ Levandose em consideração que desde o estudo original de
Janz e Christian"10 sabe-se que "transtornos psiquiátricos diagnosticáveis em senso estrito não devem ser esperados nestes pacientes", faz-se necessário o estudo da expressão dos traços de personalidade que vá além da sua classificação categorial.

A correlação entre a presença de disfunção executiva e atencional e a presença de traços de personalidade impulsivos nos pacientes com EMJ e sua relação com as variáveis clínicas ainda não foi realizada. Esta análise se faz necessária a fim de estabelecer se estes achados são coincidentes ou se inter-relacionam. Além disso, estudos abordando os aspectos neuropsicológicos e de personalidade de pacientes com EMJ, são de grande importância, não só no auxílio diagnóstico e maior compreensão deste fenômeno, mas também pela possibilidade de fornecer subsídios para diferentes abordagens terapêuticas.

Este estudo teve como objetivos: 1. verificar se as funções atencionais e executivas encontram-se prejudicadas em pacientes com EMJ e determinar se há diferentes níveis de gravidade no comprometimento destas funções; 2 . verificar se há alteração dos traços de personalidade, através de instrumento objetivo em pacientes com EMJ; 3. analisar a correlação entre o desempenho nas funções executivas e atencionais e a expressão de traços de personalidade relacionados a um pior controle de impulsos; 4. verificar se as variáveis clínicas da epilepsia se correlacionam com o desempenho cognitivo e com a expressão de um pior controle de impulsos em pacientes com EMJ.

\section{MÉTODOS}

\section{Pacientes}

Os 42 pacientes incluídos neste estudo foram avaliados no Centro de Diagnóstico e Tratamento de Epilepsia do Instituto de Psiquiatria do Hospital das Clínicas da USP, sendo classificados segundo os critérios para a classificação de síndromes epilépticas da International League Against Epilepsy. ${ }^{17}$ Vinte pacientes $(47,6 \%)$ eram do sexo masculino, com idade média de 26,57 anos [SD 8,38], 10,10 anos [SD 1,83] de escolaridade e QI estimado médio de 91,5 [SD 9,94]. Foram excluídos deste estudo pacientes com 
outras formas de epilepsia generalizada idiopática ou com diagnóstico duvidoso de EMJ; com QI estimado inferior a 80; com menos de quatro anos de escolaridade formal; que apresentassem sinais de intoxicação medicamentosa ou qualquer outra condição que pudesse provocar ou predispor prejuízo grave das funções cognitivas; com diagnóstico de transtorno psicótico; transtorno psiquiátrico grave que demandasse tratamento emergencial; que demonstrassem recusa em assinar o termo livre e esclarecido.

\section{Controles}

Foram avaliados também 54 sujeitos controles, sendo que 12 sujeitos foram excluídos pela presença de transtorno psiquiátrico. Dos 42 sujeitos incluídos, 19 (45,2\%) eram do sexo masculino, com idade média de 26,96 anos [SD 8,48], 10,61 anos [SD 2,46] de escolaridade e QI estimado médio de 94,04 [SD 7,50]. Foram excluídos sujeitos controles com QI estimado inferior a 80, com menos de quatro anos de escolaridade formal, com história de diagnóstico prévio ou atual de transtornos psiquiátricos (menos de dois anos de remissão dos sintomas) ou neurológicos, que não aceitaram assinar o termo de consentimento livre e esclarecido.

Os grupos de pacientes com EMJ e sujeitos controles foram semelhantes quanto ao gênero [ $p=0,827]$, idade $[p=0,959]$, e escolaridade [ $p=0,653]$.

\section{Instrumentos}

\section{Avaliação Neuropsicológica}

Os testes utilizados para medir o funcionamento das funções atencionais e executivas foram: Dígitos Direto $e$ Dígitos Indireto; ${ }^{18}$ Stroop Color Test (SCT) $;{ }^{19}$ Trail Making Test (TMT); ${ }^{8}$ Wisconsin Card Sorting Test (WCST);20 Matching Familiar Figures Test (MFFT) ${ }^{21}$ e Teste de Fluência Verbal. ${ }^{22}$

\section{Avaliação dos Traços de Personalidade}

A avaliação dos traços de Personalidade foi feita através do Inventário de Temperamento e Caráter (ITC). 23,24 O ITC trata-se de questionário de autopreenchimento composto por 240 questóes no molde "Verdadeiro ou Falso" e avalia a expressão dos traços de temperamento e caráter da personalidade a partir da proposta de investigação do Modelo Psicobiológico de Cloninger. Segundo Cloninger. ${ }^{25,26} \mathrm{~A}$ dimensão de Temperamento engloba os traços de determinação heterogenética, cuja expressão é apenas parcialmente afetada pelas influências ambientais e é constituída por quatro fatores Busca de Novidades (BN), Esquiva ao Dano (ED), Dependência de Gratificação (DG) e Persistência (P). Já a dimensão de Caráter engloba os traços moldados ao longo do desenvolvimento, resultantes das experiências de aprendizagem propiciadas por diferentes influências ambientais e compreende três fatores: Autodirecionamento (AD), Cooperatividade (C) e Autotranscendência (AT). No ITC, cada um dos quatro fatores da dimensão de Temperamento e dos três fatores da dimensão de Caráter (exceto Persistência) é acessado pela soma de escores em três a cinco subfatores, que medem traços mais específicos. Por exemplo, o fator de temperamento Busca por Novidades (BN) é subdividido em outros quatro subfatores: BN1 (medida de excitabilidade exploratória vs. rigidez), BN2 (medida de impulsividade vs. reflexão), BN3 (medida de extravagância vs. reserva) e BN4 (medida de desordenação vs. organização).

Sabe-se que a presença de sintomas depressivos e ansiosos pode influenciar os escores obtidos nos Fatores Esquiva ao Dano, Autodirecionamento e Cooperatividade do ITC. ${ }^{27}$ Por esta razão, aplicamos a Escala de Depressão de Beck (BDI) ${ }^{28,29}$ e o Inventário de Ansiedade TraçoEstado $^{30}$ a fim de se controlar estas variáveis.

\section{Variáveis Clínicas}

As variáveis clínicas da epilepsia analisadas foram: i. frequência das crises, diferenciando-se crises TCG, mioclônicas e ausências; ii. idade de início da epilepsia; iii. tempo entre a instalação da doença e o controle das crises através de DAE; iv. dificuldade no controle das crises epilépticas (mensurada pela quantidade de DAE necessárias); v. história familiar de epilepsia; vi. história familiar de transtornos psiquiátricos e; vii. história pessoal de transtornos psiquiátricos. Segundo as variáveis clínicas pacientes foram divididos em dois grandes grupos dependendo da sua resposta ao uso de VPA e da frequência de crises em:

I. Grupo de fácil controle (menor gravidade da epilepsia): pacientes de fácil controle das crises, sendo este obtido com doses baixas de VPA ou Divalproato de Sódio (até 1g/dia) $(\mathrm{n}=32)$.

II. Grupo de difícil controle (maior gravidade da epilepsia): pacientes com difícil controle das crises, sendo este obtido com doses elevadas de VPA ou Divalproato de Sódio (acima de 1g/dia) $(\mathrm{n}=10)$.

\section{Análise Estatística}

Uma primeira análise estatística foi empregada para a comparação do desempenho de pacientes com EMJ e controles nos testes neuropsicológicos através do teste $t$ de Student no caso de distribuição dentro da normalidade e o teste de Mann-Whitney sem distribuição normal. Em um segundo momento, foi usada a análise de covariância (ANCOVA), adotando-se o QI estimado como covariável, Os pacientes com EMJ e sujeitos controle diferiram em relação ao QI estimado [ $p=0,042]$. Estabeleceu-se um critério clínico para a avaliação do nível de gravidade da DE dos pacientes com EMJ, adotando-se como modelo o critério clínico de Rzezak et al. ${ }^{31}$ Assim, consideramos $\mathrm{DE}$ quando o score bruto em um teste do paciente foi pelo 
menos um desvio padrão inferior à média dos controles naquele teste em pelo menos dois paradigmas (Tabela 1 ).

Tabela 1. Descrição do critério clínico para avaliação da gravidade da DE

\begin{tabular}{ll}
\hline \multicolumn{1}{c}{ Presença de } & \multicolumn{1}{c}{$\begin{array}{c}\text { Score do paciente 1 SD a baixo } \\
\text { no mínimo em 2 subtestes }\end{array}$} \\
\hline DE leve & Score do paciente $1 \mathrm{SD}$ a baixo em 2 subtestes \\
DE moderada & Score do paciente 1 SD a baixo em 3 ou 4 subtestes \\
DE grave & Score do paciente 1 SD a baixo em 5 ou mais subtestes \\
\hline
\end{tabular}

Para avaliar a diferença nos escores obtidos pelos pacientes com EMJ e pelos sujeitos controles no ITC foi usada a análise de covariância (ANCOVA), adotandose os escores nas escalas de depressão e ansiedade como covariáveis, uma vez que pacientes e controles diferiram nestas medidas.

Para determinar a correlação entre o desempenho dos pacientes com EMJ nos testes neuropsicológicos e a presença de traços de personalidade relacionados a um pior controle de impulsos foi usada a análise de correlação de Pearson no caso de distribuição normal e a análise de correlação de Spearman no caso de distribuição não-normal. Com o intuito de verificar a influência das variáveis clínicas da epilepsia sobre o desempenho na bateria de testes neuropsicológicos, a presença de traços de personalidade impulsivos foram realizadas duas análises estatísticas distintas: para variáveis clínicas categoriais a análise de correlação de Pearson no caso de distribuição normal e a correlação de Spearman no caso de distribuição não-normal; para variáveis clínicas qualitativas foi utilizado o teste $t$ de Student.

Por fim, a comparação entre os grupos de fácil e difícil controle da epilepsia foi realizada através do teste t de Student no caso de distribuição normal e do teste de MannWhitney no caso de distribuição não-normal.

\section{RESULTADOS}

\section{Avaliação Neuropsicológica}

Da avaliação do desempenho dos pacientes com EMJ e dos sujeitos controles nos testes avaliadores das funções executivas e atencionais (FE) os pacientes com EMJ tiveram pior desempenho do que os sujeitos controles nos seguintes testes: Dígitos Diretos; Dígitos Indiretos; no tempo de execução do Stroop Color Test 1, 2 e Stroop 3; no número de erros no Stroop Color Test 2 e 3; no tempo de execução do Trail Making Test A e Trail Making Test B; no número de erros no Trail Making Test B; Fluência Verbal; Wisconsin Card Sorting Test (número de categorias, número total de erros, número de erros perseverativos, número de respostas perseverativas e perdas de set) e no número de erros no Matching Familiar Figures Test, nas duas análises realizadas (Tabela 2).

Tabela 2. Média e desvio padrão do desempenho nos testes de FE e atencionais de pacientes com EMJ e controles.

\begin{tabular}{|c|c|c|c|c|}
\hline Teste & $\begin{array}{c}\text { EMJ } \\
(n=42)\end{array}$ & $\begin{array}{c}\text { Controles } \\
(\mathrm{N}=42)\end{array}$ & $\begin{array}{c}p \\
\text { (teste t e Mann Whitney) }\end{array}$ & $\begin{array}{c}p \\
\text { (ANCOVA) }\end{array}$ \\
\hline DD (WAIS-R) & $5,09 \pm 1,73$ & $7,02 \pm 1,90$ & $0,0011^{\text {a }}$ & $0,001^{\mathrm{c}}$ \\
\hline DI (WAIS-R) & $3,61 \pm 1,56$ & $5,35 \pm 1,84$ & $0,001^{\text {a }}$ & $0,001^{\mathrm{c}}$ \\
\hline SCT - 1 tempo & $21,78 \pm 5,71$ & $14,21 \pm 2,78$ & $0,001^{b}$ & $0,001^{\mathrm{c}}$ \\
\hline SCT - 1 erros & $0,03 \pm 0,15$ & $0,02 \pm 0,11$ & $0,956^{\mathrm{a}}$ & $0,993^{\mathrm{c}}$ \\
\hline SCT - 2 tempo & $26,61 \pm 8,0$ & $17,68 \pm 4,22$ & $0,001^{b}$ & $0,001^{c}$ \\
\hline SCT - 2 erros & $0,16 \pm 0,0$ & $0,43 \pm 0,00$ & $0,012^{b}$ & 0,030 c \\
\hline SCT - 3 tempo & $36,73 \pm 10,72$ & $23,80 \pm 5,71$ & $0,001^{b}$ & $0,001^{\mathrm{c}}$ \\
\hline SCT - 3 erros & $1,90 \pm 0,23$ & $1,87 \pm 0,57$ & $0,001^{b}$ & $0,001^{\mathrm{c}}$ \\
\hline TMT - A tempo & $39,59 \pm 10,96$ & $29,21 \pm 9,75$ & $0,001^{\mathrm{a}}$ & $0,001^{\mathrm{c}}$ \\
\hline TMT - A erros & $0,09 \pm 0,29$ & $0,04 \pm 0,21$ & $0,403^{a}$ & $0,541^{\mathrm{c}}$ \\
\hline TMT - B tempo & $73,71 \pm 27,32$ & $57,76 \pm 24,75$ & $0,006^{\mathrm{a}}$ & $0,006^{c}$ \\
\hline TMT - B erros & $0,80 \pm 1,04$ & $0,14 \pm 0,41$ & $0,001^{b}$ & $0,001^{\mathrm{c}}$ \\
\hline FAS - letra F & $9,19 \pm 2,22$ & $13,97 \pm 3,97$ & $0,001^{b}$ & $0,001^{\mathrm{c}}$ \\
\hline FAS - letra A & $8,52 \pm 2,22$ & $12,35 \pm 3,53$ & $0,001^{b}$ & $0,001^{\mathrm{c}}$ \\
\hline FAS - letra S & $7,97 \pm 2,53$ & $12,33 \pm 3,72$ & $0,001^{b}$ & $0,001^{\mathrm{c}}$ \\
\hline FAS - total & $25,69 \pm 5,00$ & $38,66 \pm 9,72$ & $0,001^{b}$ & $0,001^{\mathrm{c}}$ \\
\hline WCST (categ.) & $2,30 \pm 1,17$ & $3,76 \pm 1,16$ & $0,001^{b}$ & $0,001^{\mathrm{c}}$ \\
\hline WCST (ET) & $21,02 \pm 9,75$ & $8,11 \pm 4,24$ & $0,001^{b}$ & $0,001^{\mathrm{c}}$ \\
\hline WCST (EP) & $11,28 \pm 5,37$ & $6,07 \pm 3,67$ & $0,001^{a}$ & $0,001^{\mathrm{c}}$ \\
\hline WCST (RP) & $13,48 \pm 6,82$ & $9,76 \pm 4,63$ & $0,005^{b}$ & $0,009^{c}$ \\
\hline WCST (PS) & $1,43 \pm 0,97$ & $0,33 \pm 0,61$ & $0,001^{a}$ & $0,001^{\mathrm{c}}$ \\
\hline MFFT - erros & $9,64 \pm 3,71$ & $6,78 \pm 4,08$ & $0,011^{a}$ & $0,002^{\mathrm{c}}$ \\
\hline MFFT - tempo & $372,28 \pm 89,34$ & $343,92 \pm 105,57$ & $0,188^{\mathrm{a}}$ & $0,165^{\mathrm{c}}$ \\
\hline
\end{tabular}

${ }^{\mathrm{a}}$ Teste de Mann-Whitney. ${ }^{\mathrm{b}}$ Teste t de Student. ${ }^{\mathrm{c}}$ Ancova controlando por QI.

DD: Dígitos Diretos; DI: Dígitos Indiretos; SCT: Stroop Color Test; TMT: Trail Making Test; FAS: Teste de Fluência Verbal WCST: Wisconsin Card Sorting Test; WCST (categ): Wisconsin Card Sorting Test (número de categorias); WCST (EP): Wisconsin Card Sorting Test (erros perseverativos); WCST (RP): Wisconsin Card Sorting Test (respostas perseverativas); WCST (QS): Wisconsin Card Sorting Test (perda de set); MFFT: Matching Familiar Figures Test. 
Da avaliação da gravidade da Disfunção Executiva (DE) a partir da comparação do desempenho dos pacientes com EMJ e médias dos escores dos controles nos testes de FE, observou-se que $95,23 \%$ dos pacientes apresentaram DE sendo que dos 42 pacientes avaliados, 35 (83,33\%) apresentaram DE moderada ou grave.

\section{Avaliação dos Traços de Personalidade}

\section{Dimensão de Temperamento}

Na comparação dos resultados entre pacientes com EMJ e controles da dimensão de Temperamento do ITC, foram encontradas diferenças estatisticamente significantes entre os grupos nos subfatores Busca por Novidades; Esquiva ao Dano e Dependência de Gratificação 4 (Tabela 3).

\section{Dimensão de Caráter}

Na comparação dos resultados da dimensão de Caráter do ITC, foram encontradas diferenças estatisticamente significantes entre os grupos nos subfatores: Autodirecionamento e Cooperatividade (Tabela 4).

Correlação entre o desempenho nas funções executivas e atencionais e a expressão de traços de personalidade relacionados a um pior controle de impulsos

Como medida da expressão de traços de personalidade impulsivos utilizamos os escores no Fator Busca por Novidades do ITC, sendo que quanto maior a pontuação em BN, menor o controle de impulsos. Os escores no fator Busca por Novidades foram correlacionados com os

Tabela 3. Média e desvio padrão dos escores na dimensão de Temperamento do ITC de pacientes com EMJ e controles.

\begin{tabular}{|c|c|c|c|}
\hline Dimensão de Temperamento & $\begin{array}{c}\text { EMJ } \\
(n=42)\end{array}$ & $\begin{array}{c}\text { Controles } \\
(n=42)\end{array}$ & $p$ \\
\hline Busca por Novidades total (BN) & $24,45 \pm 3,5$ & $18,16 \pm 4,33$ & $0,001^{\mathrm{c}}$ \\
\hline BN1 - Excitabilidade Exploratória vs. Rigidez & $7,42 \pm 1,25$ & $5,61 \pm 2,07$ & $\mathbf{0 , 0 0 1}{ }^{\mathrm{c}}$ \\
\hline BN2 - Impulsividade vs. Reflexão & $6,26 \pm 1,36$ & $3,54 \pm 1,62$ & $\mathbf{0 , 0 0 1}{ }^{\mathrm{c}}$ \\
\hline BN3 - Extravagância vs. Reserva & $5,28 \pm 1,62$ & $4,50 \pm 2,06$ & $0,898^{\mathrm{c}}$ \\
\hline BN4 - Desordenação vs. Organização & $5,47 \pm 1,61$ & $4,50 \pm 1,86$ & $0,010^{\mathrm{c}}$ \\
\hline Esquiva ao Dano total (ED) & $21,92 \pm 3,52$ & $16,45 \pm 6,24$ & $0,002^{\mathrm{c}}$ \\
\hline ED1 - Preocupação antecipatória vs. Otimismo & $7,07 \pm 1,82$ & $4,16 \pm 2,28$ & $\mathbf{0 , 0 0 1}{ }^{\mathrm{c}}$ \\
\hline ED2 - Medo da incerteza vs. Confiança & $5,14 \pm 1,31$ & $4,71 \pm 1,55$ & $0,906^{\mathrm{c}}$ \\
\hline ED3 - Timidez vs. Sociabilidade & $4,95 \pm 1,52$ & $4,38 \pm 1,98$ & $0,845^{\mathrm{c}}$ \\
\hline ED4 - Fadigabilidade vs. Astenia & $4,73 \pm 1,41$ & $3,23 \pm 2,04$ & $0,004^{c}$ \\
\hline Dependência de Gratificação total (DG) & $14,11 \pm 2,42$ & $15,19 \pm 3,1$ & $0,288^{\mathrm{c}}$ \\
\hline DG1 - Sentimentalismo vs. Insensibilidade & $6,73 \pm 1,41$ & $6,47 \pm 1,85$ & $0,774^{\mathrm{c}}$ \\
\hline DG3 - Apego vs. Desapego & $4,85 \pm 1,55$ & $5,07 \pm 1,77$ & $0,810^{\mathrm{c}}$ \\
\hline DG4 - Dependência vs. Independência & $2,52 \pm 1,29$ & $3,61 \pm 1,46$ & $0,041^{\mathrm{c}}$ \\
\hline Persistência $(\mathrm{P})$ & $5,30 \pm 1,48$ & $4,73 \pm 1,65$ & $0,314^{\mathrm{c}}$ \\
\hline
\end{tabular}

cAncova controlando por Beck, IDATE-E e IDATE-T.

Tabela 4. Média e desvio padrão dos escores na dimensão de Temperamento do ITC de pacientes com EMJ e controles.

\begin{tabular}{|c|c|c|c|}
\hline Dimensão de Caráter & $\begin{array}{c}\text { EMJ } \\
(n=42)\end{array}$ & $\begin{array}{c}\text { Controles } \\
(n=42)\end{array}$ & $p$ \\
\hline Autodirecionamento total (AD) & $24,47 \pm 4,69$ & $31,59 \pm 7,12$ & $0,001^{\mathrm{c}}$ \\
\hline AD1 - Responsabilidade vs. Atribuição de culpa & $4,83 \pm 1,98$ & $5,69 \pm 1,77$ & $0,639^{\mathrm{c}}$ \\
\hline AD2 - Determinação vs. Metas não objetivas & $3,85 \pm 1,55$ & $5,83 \pm 2,07$ & $0,001^{\mathrm{c}}$ \\
\hline AD3 - Desembaraço vs. Apatia & $6,19 \pm 1,53$ & $7,21 \pm 2,22$ & $0,088^{\mathrm{c}}$ \\
\hline AD4 - Autoaceitação vs. Autorrecusa & $6,21 \pm 0,33$ & $7,33 \pm 0,51$ & $0,106^{\mathrm{c}}$ \\
\hline AD5 - Segunda Natureza congruente & $7,11 \pm 1,78$ & $9,19 \pm 2,03$ & $0,002^{c}$ \\
\hline Cooperatividade total (C) & $30,40 \pm 4,61$ & $33,78 \pm 4,73$ & $0,113^{\mathrm{c}}$ \\
\hline C1 - Aceitação social vs. Intolerância & $6,33 \pm 1,35$ & $6,61 \pm 1,20$ & $0,268^{\mathrm{c}}$ \\
\hline C2 - Empatia vs. Desinteresse Social & $5,16 \pm 1,12$ & $5,45 \pm 1,23$ & $0,750^{\mathrm{c}}$ \\
\hline C3 - Utilidade vs. Inutilidade & $5,28 \pm 1,58$ & $6,30 \pm 1,38$ & $0,200^{\mathrm{c}}$ \\
\hline C4 -Compaixão vs. Vingança & $7,69 \pm 1,71$ & $8,42 \pm 1,41$ & $0,400^{\mathrm{c}}$ \\
\hline C5 - Generosidade vs. Egoísmo & $5,92 \pm 1,25$ & $6,97 \pm 1,47$ & $0,029^{\mathrm{c}}$ \\
\hline Autotranscendência total (AT) & $17,83 \pm 4,42$ & $15,33 \pm 6,52$ & $0,053^{\mathrm{c}}$ \\
\hline AT1 - Altruísmo vs. Autoconsciência & $6,40 \pm 1,97$ & $4,90 \pm 2,88$ & $0,086^{\mathrm{c}}$ \\
\hline AT2 - Identificação Transpessoal & $5,11 \pm 1,74$ & $3,41 \pm 0,54$ & $0,059^{\mathrm{c}}$ \\
\hline AT3 - Aceitação Espiritual vs. Materialismo & $6,30 \pm 2,45$ & $7,16 \pm 2,38$ & $0,338^{\mathrm{c}}$ \\
\hline
\end{tabular}

c Ancova controlando por Beck, IDATE-E e IDATE-T. 
desempenhos dos pacientes com EMJ nos testes de Funções Executivas e Atencionais. Os resultados se encontram na Tabela 5.

Tabela 5. Influência dos escores em Busca por Novidades do ITC sobre o desempenho nos testes de FE nos pacientes com EMJ.

\begin{tabular}{lc}
\hline & Busca por Novidades \\
\hline DD (WAIS-R) & $\mathbf{0 , 0 0 1}$ \\
DI (WAIS-R) & $\mathbf{0 , 0 0 4 ^ { \mathrm { e } }}$ \\
SCT - 1 tempo & $0,084^{\mathrm{d}}$ \\
SCT - 1 erros & $0,660^{\mathrm{d}}$ \\
SCT - 2 tempo & $\mathbf{0 , 0 3 8}^{\mathrm{e}}$ \\
SCT - 2 erros & $0,628^{\mathrm{d}}$ \\
SCT - 3 tempo & $0,128^{\mathrm{d}}$ \\
SCT - 3 erros & $0,187^{\mathrm{d}}$ \\
TMT - A tempo & $\mathbf{0 , 0 0 3}$ \\
TMT - A erros & $0,678^{\mathrm{d}}$ \\
TMT - B tempo & $\mathbf{0 , 0 0 7 ^ { \mathrm { e } }}$ \\
TMT - B erros & $\mathbf{0 , 0 2 0 ^ { \mathrm { e } }}$ \\
FAS - total & $0,342^{\mathrm{d}}$ \\
WCST (categ.) & $\mathbf{0 , 0 2 4 ^ { \mathrm { e } }}$ \\
WCST (ET) & $\mathbf{0 , 0 4 6}^{\mathrm{e}}$ \\
WCST (EP) & $0,107^{\mathrm{d}}$ \\
WCST (RP) & $0,158^{\mathrm{d}}$ \\
WCST (PS) & $\mathbf{0 , 0 1 1}^{\mathrm{e}}$ \\
MFFT - erros & $0,240^{\mathrm{d}}$ \\
MFFT - tempo & $0,983^{\mathrm{d}}$ \\
\hline
\end{tabular}

${ }^{\mathrm{d}}$ Correlação de Pearson (p). ${ }^{\mathrm{e}}$ Correlação de Spearman (p).

\section{Comparação dos Grupos de Fácil e Difícil controle da Epilepsia}

\section{Avaliação neuropsicológica}

Os grupos I (fácil controle da epilepsia) e II (difícil controle da epilepsia) de pacientes com EMJ foram comparados quanto aos seus desempenhos nos testes de FE. Os resultados se encontram na Tabela 6.

\section{Avaliação dos Traços de Personalidade}

Da comparação dos grupos de fácil e difícil controle da epilepsia nos escores no Fator Busca por Novidades do ITC, medida usada para mensurar a expressão de traços de personalidade impulsivos, observamos que o grupo II apresentou maior expressão dos traços de personalidade impulsivos do que o grupo I (Tabela 7).

\section{DISCUSSÃO}

Os nossos resultados corroboraram os estudos anteriores que identificaram disfunção executiva nos pacientes com EMJ.2-6 Entretanto, devido ao uso de uma bateria mais ampla, este trabalho pode detalhar quais aspectos das funções executivas e das funções atencionais, frequentemente negligenciadas, encontram-se prejudicados. Desta forma, este estudo demonstrou que pacientes com EMJ apresentam déficits específicos das capacidades
Tabela 6. Comparação do desempenho dos grupos I e II de pacientes com EMJ nos testes de FE.

\begin{tabular}{|c|c|c|c|}
\hline Teste & $\begin{array}{c}\text { Grupo I }(n=32) \\
\mu \pm \text { SD }\end{array}$ & $\begin{array}{c}\text { Grupo II }(n=10) \\
\mu \pm \text { SD }\end{array}$ & $p$ \\
\hline DD (WAIS-R) & $5,62 \pm 1,56$ & $3,40 \pm 1,07$ & $0,001^{a}$ \\
\hline DI (WAIS-R) & $4,00 \pm 1,58$ & $2,40 \pm 0,51$ & $0,001^{\mathrm{a}}$ \\
\hline SCT - 1 tempo & $20,40 \pm 5,13$ & $26,2 \pm 5,43$ & $0,013^{b}$ \\
\hline SCT - 1 erros & $0,03 \pm 0,76$ & $0,01 \pm 0,01$ & $0,896^{\mathrm{a}}$ \\
\hline SCT - 2 tempo & $24,5 \pm 6,4$ & $33,4 \pm 9,03$ & $0,013^{b}$ \\
\hline SCT - 2 erros & $0,09 \pm 0,29$ & $0,40 \pm 0,69$ & $0,207^{\mathrm{b}}$ \\
\hline SCT - 3 tempo & $33,62 \pm 10,35$ & $49,9 \pm 4,84$ & $0,006^{b}$ \\
\hline SCT - 3 erros & $1,46 \pm 1,39$ & $3,30 \pm 2,54$ & $0,052^{b}$ \\
\hline TMT - A tempo & $36,67 \pm 10,35$ & $49,9 \pm 4,84$ & $0,001^{\text {a }}$ \\
\hline TMT - A erros & $0,09 \pm 0,29$ & $0,10 \pm 0,31$ & $0,957^{\mathrm{a}}$ \\
\hline TMT - B tempo & $70,46 \pm 28,96$ & $84,10 \pm 18,79$ & $0,008^{a}$ \\
\hline TMT - B erros & $0,40 \pm 0,61$ & $2,11 \pm 1,10$ & $0,001^{b}$ \\
\hline FAS - letra F & $9,90 \pm 1,90$ & $6,90 \pm 1,52$ & $\mathbf{0 , 0 0 1}{ }^{\mathrm{b}}$ \\
\hline FAZ - letra A & $8,87 \pm 2,04$ & $7,41 \pm 2,52$ & $0,114^{\mathrm{b}}$ \\
\hline FAZ - letra S & $8,09 \pm 2,06$ & $7,62 \pm 2,06$ & $0,548^{\mathrm{b}}$ \\
\hline FAS - total & $26,87 \pm 4,51$ & $21,90 \pm 4,81$ & $0,012^{b}$ \\
\hline WCST (categ.) & $2,62 \pm 1,07$ & $1,30 \pm 0,94$ & $0,002^{b}$ \\
\hline WCST (ET) & $18,21 \pm 8,39$ & $30,0 \pm 8,57$ & $0,002^{\mathrm{b}}$ \\
\hline WCST (EP) & $10,90 \pm 5,30$ & $13,60 \pm 5,08$ & $0,123^{\mathrm{a}}$ \\
\hline WCST (RP) & $12,53 \pm 6,28$ & $18,0 \pm 7,36$ & $0,054^{b}$ \\
\hline WCST (PS) & $1,20 \pm 0,80$ & $2,44 \pm 0,88$ & $0,002^{b}$ \\
\hline MFFT - erros & $8,46 \pm 2,97$ & $13,14 \pm 3,43$ & $0,001^{\mathrm{a}}$ \\
\hline MFFT - tempo & $394,00 \pm 86,18$ & $317,7 \pm 77,27$ & $0,045^{\text {a }}$ \\
\hline
\end{tabular}

${ }^{a}$ Teste de Mann-Whitney. ${ }^{\mathrm{b}}$ Teste $\mathrm{t}$ de Student. $\mu$ : média. SD: desvio padrão; DD: Dígitos Diretos; DI: Dígitos Indiretos; SCT: Stroop Color Test; TMT: Trail Making Test; FAS: Teste de Fluência Verbal; WCST: Wisconsin Card Sorting Test; WCST (categ): Wisconsin Card Sorting Test (número de categorias); WCST (EP): Wisconsin Card Sorting Test (erros perseverativos); WCST (RP): Wisconsin Card Sorting Test (respostas perseverativas); WCST (QS): Wisconsin Card Sorting Test (perda de set); MFFT: Matching Familiar Figures Test.

Tabela 7. Comparação do desempenho dos grupos I e II de pacientes com EMJ no fator Busca por Novidades do ITC.

\begin{tabular}{lccc}
\hline & $\begin{array}{c}\text { Grupo I }(\mathbf{n}=\mathbf{3 2}) \\
\boldsymbol{\mu} \pm \mathbf{S D}\end{array}$ & $\begin{array}{c}\text { Grupo II }(\mathbf{n}=\mathbf{1 0}) \\
\boldsymbol{\mu} \pm \mathbf{S D}\end{array}$ & $\boldsymbol{p}$ \\
\hline $\begin{array}{l}\text { Busca por } \\
\text { novidades }\end{array}$ & $23,84 \pm 3,74$ & $26,40 \pm 1,42$ & $\mathbf{0 , 0 0 3}^{\text {b }}$ \\
\hline${ }^{\mathrm{b}}$ Teste t de Student. $\mu$ : média. SD: desvio padrão. & &
\end{tabular}

de memória operativa, controle inibitório, formação de conceitos, manutenção de metas, flexibilidade mental e fluência verbal. Quanto à atenção, observamos falhas desde os processos mais básicos da atenção como o alerta e o span atencional até os processos mais complexos que exigem atenção dividida e sustentada. Observamos também que embora a disfunção executiva estivesse presente na maior parte dos pacientes, havia diferentes níveis de prejuízo. A gravidade da disfunção executiva nos pacientes com EMJ não foi avaliada previamente. Neste estudo, utilizamos o critério clínico de Rzezak et al. ${ }^{31}$ com o qual determinamos que $83,33 \%$ dos pacientes com EMJ apresentavam disfunção executiva moderada ou grave. Desta forma, é importante ressaltar que além das diferenças qualitativas, há diferenças quantitativas no comprometimento cognitivo 
destes pacientes. Embora, a maior parte destes pacientes tenha disfunção executiva/atencional, esta se apresenta com intensidades diferentes. Portanto, espera-se que o impacto desta disfunção seja desigual. Nossos achados indicam a necessidade da utilização de baterias amplas de testes neuropsicológicos para avaliar as funções executivas/ atencionais e de um critério clínico para classificar os níveis de disfunção executiva em pacientes com EMJ, como já demonstrado previamente no estudo de crianças com epilepsia do lobo temporal. ${ }^{31}$

Nosso estudo corrobora ainda a presença de traços de personalidade impulsivos nos pacientes com EMJ, demonstrado anteriormente. ${ }^{10-16} \mathrm{O}$ uso do ITC nos permitiu pormenorizar o perfil impulsivo destes pacientes caracterizado por maior necessidade de buscar novidades, dificuldade em manter rotinas assim como dificuldades na capacidade de organização para atingir metas. Além disso, estes pacientes apresentam uma baixa tolerância à frustração e rápida tendência ao desinteresse frente a uma situação aversiva. Apresentam ainda uma tendência maior à fadiga, com prejuízo do span atencional durante uma atividade prolongada. Os pacientes com EMJ apresentaram uma maior dificuldade de estabelecer metas a longo prazo, sendo levados pelos impulsos imediatos corroborando observações clínicas prévias sobre estes pacientes.

A avaliação dos traços de personalidade impulsivos e do desempenho nos testes de funções executivas e atencionais demonstrou que pacientes com pior controle dos impulsos também apresentam piores desempenhos nos testes avaliadores das funções executivas e atencionais, com prejuízos extensos destas funções. Mostraram-se prejudicadas as capacidades de span atencional, controle mental, controle inibitório, atenção sustentada e dividida, automonitorização, formação de conceitos, flexibilidade mental e manutenção de metas. Esta relação provê evidências clínicas sobre a presença de disfunção do lobo frontal nos pacientes com EMJ. Estas evidências clínicas são corroboradas pelos achados de estudos neuropatológicos ${ }^{32}$ e estudos de neuroimagem estrutural e funcional em pacientes com EMJ. ${ }^{33-37}$

Além disso, verificamos a existência de dois grupos distintos de pacientes, sendo que pacientes mais refratários apresentaram pior disfunção executiva e maior expressão dos traços de personalidade impulsivos. Este é o primeiro estudo a delinear perfis distintos dentro do grupo de pacientes com EMJ. O conjunto de achados de pior funcionamento cognitivo e pior controle dos impulsos nos pacientes com difícil controle da epilepsia pode sugerir a presença de endofenótipos clínicos distintos. Há evidências pungentes de que as comorbidades psiquiátricas assim como as alterações cognitivas sejam epifenômenos de alterações estruturais e funcionais existentes, que são também determinantes da maior gravidade da epilepsia. A necessidade de melhor caracterização fenotípica dos pacientes com EMJ a fim de incluir endofenótipos clínicos pode auxiliar na abordagem destes pacientes, assim como nos estudos genéticos e de neuroimagem da síndrome, na tentativa de elucidar as bases neurobiológicas desta doença.

\section{REFERÊNCIAS}

1. Dreifuss FE. Juvenile myoclonic epilepsy: characteristics of a primary generalized epilepsy. Epilepsia 1989;30(S4):1-7.

2. Piazzini A, Turner K, Vignoli A, Canger R, Canevini MP. Frontal cognitive dysfunction in juvenile myoclonic epilepsy. Epilepsia 2008 Apr;49(4):657-62.

3. Pascalicchio TF, de Araujo Filho GM, da Silva Noffs MH, Lin K, Caboclo LO, Vidal-Dourado M, Ferreira Guilhoto LM, Yacubian EM. Neuropsychological profile of patients with juvenile myoclonic epilepsy: a controlled study of 50 patients. Epilepsy Behav 2007 Mar;10(2):263-7. Epub 2007 Jan 26.

4. Sonmez F, Atakli D, Sari H, Atay T, Arpaci B. Cognitive function in juvenile myoclonic epilepsy. Epilepsy and Behavior 2004 Jun;5(3): 329-36.

5. Devinsky O, Gershengorn J, Brown E. et al. Frontal functions in juvenile myoclonic epilepsy. Journal of Neurology, Neurosurgery and Psychiatry 1997;10:243-6.

6. Swartz BE, Simpkins F, Halgren E. et al. Visual working memory in primary generalized epilepsy: an ${ }^{18}$ FDG-PET study. Neurology 1996;5:1203-12.

7. Malloy-Diniz LF, Leite WB, Moraes PHP, Correa H, Bechara A, Fuentes D. Brazilian Portuguese version of the Iowa Gambling Task: transcultural adaptation and discriminant validity. Revista Brasileira de Psiquiatria (São Paulo) 2008;144.

8. Lezak MD. Neuropychological Assessment. $4^{\text {th }}$ ed. New York: Oxford University Press; 2005.

9. Fuster JM. Cognitive functions of the frontal lobes. In Miller BL, Cummings JL (eds.). The human frontal lobes: Functions and disorders. New York: Guilford Press; 1999.

10. Janz D, Christian W. Impulsiv petit-mal. Deutsche Z. Nervenheilk 1957;176:346-86.

11. Perini GI, Tosin C, Carraro C, Bernasconi G, Canevini MP, Canger R, Pellegrini A, Testa G. Interictal mood and personality disorders in temporal lobe epilepsy and juvenile myoclonic epilepsy. J Neurol Neurosurg Psychiatry 1996 Dec;61(6):601-5.

12. Ritaccio AL, Devinsky O. Personality disorders in epilepsy. In: Eltinger AB, Kanner AM (eds.). Psychiatric issues in epilepsy: a practical guide to diagnosis and treatment. Lippincott Williams \& Wilkins; 2001. p. 147-61.

13. Gelisse P, Genton P, Samuelian JC, Thomas P, Bureau M. Psychiatric disorders in juvenile myoclonic epilepsy. Revue Neurologique (Paris) 2001 Mar;157(3):297-302.

14. Trinka E, Kienpointner G, Unterberger I, Luef G, Bauer G, Doering LB, Doering S. Psychiatric comorbidity in juvenile myoclonic epilepsy. Epilepsia 2006 Dec;47(12):2086-91.

15. De Araújo Filho GM, Pascalicchio TF, Sousa PS, Lin K, Ferreira Guilhoto LM, Yacubian EM. Psychiatric disorders in juvenile myoclonic epilepsy: a controlled study of 100 patients. Epilepsy Behav. 2007 May; 10(3):437-41.

16. Plattner B, Pahs G, Kindler J, Williams RP, Hall RE, Mayer H, Steiner H, Feucht M. Juvenile myoclonic epilepsy: a benign disorder? Personality traits and psychiatric symptoms. Epilepsy and Behavior 2007 Jun;10(4):560-4.

17. ILAE - Commission on Classification and Terminology of the International League Against Epilepsy. Proposal for Revised Classification of Epilepsy and Epileptic Syndrome. Epilepsia 1989;30:389-99.

18. Wechsler D. WAIS-R Manual. New York: The Psychological Corporation; 1981.

19. Stroop JR. Studies of interference in serial verbal reactions. Journal of Experimental Psychology 1935;18:643-61. 
20. Heaton RK, Chelune GJ, Talley JL, Kay GK, Curtiss G. The Wisconsin Card Sorting Test Manual. Psychological Assesment Resources (Odessa) 1981.

21. Spreen O, Strauss E. A compendium of Neurospsychological Tests, Administration, Norms and Commentary. New York: Oxford University Press; 1991.

22. Spreen O, Benton. Control Oral Word Association. In: Spreen O, Strauss E. A compendium of Neurospsychological Tests, Administration, Norms and Commentary. New York: Oxford University Press; 1991.

23. Cloninger CR, Svrakic DM, Przybeck TR. A psychobiological model of temperament and character. Archives of General Psychiatry 1993 Dec;50(12):975-90.

24. Fuentes D. Jogo patológico: análise por neuroimagem, neuropsicológica e de personalidade [tese]. São Paulo: Departamento de Psiquiatria da Universidade de São Paulo; 2004.

25. Cloninger CR. A practical way to diagnosis personality disorder: a proposal. Journal of Personality Disorders 2000;14:99-108.

26. Cloninger CR. Assessment of the impulsive-compulsive spectrum of behavior by the seven-factor model of temperament and character. In: Oldham JM, Hollander E, Skodol AE (eds.). Impulsivity and compulsivity. Washington DC: American Psychiatric Press; 1996.

27. Svrakic DM, Draganic S, Hill K, Bayon C, Przybeck TR, Cloninger CR. Temperament, character, and personality disorders: etiologic, diagnostic, treatment issues. Acta Psychiatr Scand 2002;106: 189-95.

28. Beck AT, Ward CH, Mendelson M, Mock J, Erbaugh G. An inventory for for measuring depression. Archives of General Psychiatry 1961;4:53-63.

29. Gorenstein C, Andrade L, Vieira Filho AHG, Tung Teng C, Artes R. Psychometric properties of the portuguese version of the beck depression inventory on brazilian college students. Journal of Clinical Psycology 1999;55(5):553-62.
30. Spielberger CD, Gorsuch RL, Lushene RD. Manual for the State-Trait Anxiety Inventory. Palo Alto, CA: Consulting Psychologists Press; 1970.

31. Rzezak P, Fuentes D, Guimarães CA, Thome-Souza S, Kuczynski E, Li LM, Franzon RC, Leite CC, Guerreiro M, Valente KD. Frontal lobe dysfunction in children with temporal lobe epilepsy. Pediatr Neurol 2007 Sept;37(3):176-85.

32. Meencke HJ, Janz D. Neuropathological findings in primary generalized epilepsy:a study of eight cases. Epilepsia 1984;25:8-21.

33. Koepp MJ, Richardson MP, Brooks DJ, Cunningham VJ, Duncan JS. Central benzodiazepine/gamma-aminobutyric acid A receptors in idiopathic generalized epilepsy: an [11C]flumazenil positron emission tomography study. Epilepsia. 1997 Oct;38(10):1089-97.

34. Woermann FG, MC Lean MA, Barlett PA, Parker GJ, Barker GJ, Duncan JS. Short echo time single-voxel ${ }^{1} \mathrm{H}$ magnetic resonance spectroscopy in magnetic resonance imaging-negative temporal lobe epilepsy: different biochemical profile compared with hipocampal sclerosis. Ann Neurol 1999;45:369-76.

35. Savic I, Lekvall A, Greitz D, Helms G. Mr Spectroscopy shows reduced frontal lobe concentrations of $\mathrm{N}$-acetyl aspartate in patients with juvenile myoclonic epilepsy. Epilepsia 2000;41(3):290-6.

36. Mory SB, Li LM, Guerreiro CAM, Cendes F. Thalamic dysfunction juvenile myoclonic epilepsy: a proton MRS study. Epilepsia 2003;44:1402-5.

37. Meschaks A, Lindstrom P, Halldin C, Farde L, Savic I. Regional reductions in serotonin $1 \mathrm{~A}$ receptor binding in juvenile myoclonic epilepsy. Arch Neurol 2005 June;62 (6):946-50.

Endereço para correspondência:

Kette D. Valente

Rua Jesuíno Arruda, 901/51

CEP 04532-082, São Paulo, SP, Brasil

Telefone/Fax: (11) 3079-5493

E-mail: <kettevalente@msn.com> 